\title{
Polyethylene Glycol Plus Linaclotide is an Effective Bowel Preparation Regimen for Patients with Bristol Stool Scale 1-2: a Randomized Controlled Trial
}

\author{
Kai Zhao \\ Tongji Hospital \\ Ge Wang \\ Tongji Hospital \\ Suhong Xia \\ Tongji Hospital \\ Jingwen Wu \\ Tongji Hospital \\ Wangdong Zhou \\ Tongji Hospital \\ Mingyu Zhang \\ Tongji Hospital \\ Mei Liu \\ Tongji Hospital \\ Dean Tian \\ Tongji Hospital \\ Jiazhi Liao ( $\nabla$ liaojiazhi@tjh.tjmu.edu.cn ) \\ Tongji Hospital
}

\section{Research Article}

Keywords: Bowel preparation, Bristol stool form, polyethylene glycol, linaclotide

Posted Date: September 23rd, 2021

DOI: https://doi.org/10.21203/rs.3.rs-896828/v1

License: (c) (i) This work is licensed under a Creative Commons Attribution 4.0 International License.

Read Full License 
Polyethylene glycol plus Linaclotide is an effective bowel preparation regimen for patients with Bristol stool scale 1-2: a randomized controlled trial

Kai Zhao ${ }^{1}$, Ge Wang ${ }^{1}$, Suhong Xia ${ }^{1}$, Jingwen $\mathrm{Wu}^{1}$, Wangdong Zhou ${ }^{1}$, Mingyu Zhang ${ }^{1}$, Mei Liu ${ }^{1}$, Dean $\operatorname{Tian}^{1}$ and Jiazhi Liao ${ }^{1, *}$

${ }^{1}$ Department of Gastroenterology, Tongji Hospital of Tongji Medical College, Huazhong university of Science and Technology, Wuhan 430030 Hubei Province,

China

*Corresponding: liaojiazhi@tjh.tjmu.edu.cn

Full list of author information is available at the end of the article.

\section{Abstract}

Background: To evaluate the effect of using linaclotide as an adjunctive agent with $3 \mathrm{~L}$ polyethylene glycol (PEG) for bowel preparation among patients with Bristol stool form (BSFS) 1-2.

Methods: Patients with BSFS 1-2 randomly received either 3L PEG (group A) or linaclotide plus 3L PEG (group B), patients with BSFS 3-7 received 3L PEG (group C) for bowel preparation. The primary outcome was the rate of adequate bowel preparation. The secondary endpoints were the polyp detection rate (PDR), acceptability and tolerability.

Results: A total of 315 patients who underwent colonoscopy were enrolled. In perprotocol analysis, patient in group B attained significantly satisfactory bowel preparation rate than group A $\left(77.9 \%\right.$ vs $\left.61.8 \%, P_{\mathrm{AB}}=0.049\right)$. The PDR in group $\mathrm{B}$ was significantly higher than group $\mathrm{A}\left(32.7 \%\right.$ vs $\left.15.7 \%, P_{\mathrm{AB}}=0.004\right)$, especially in rectosigmoid $\left(25.0 \%\right.$ vs $\left.8.8 \%, P_{\mathrm{AB}}=0.002\right)$.

Conclusion: 3L PEG plus 290ug Linaclotide can significantly improve the quality of bowel preparation and the colorectal polyp detection rate in patients with BSFS 1-2. The bowel preparation regimen is an effective and well-tolerated bowel preparation regimen.

Trial registration: The study was registered at Chinese ClinicalTrials.gov (ChiCTR2100050437, 27/8/2021) 
Keywords: Bowel preparation, Bristol stool form, polyethylene glycol, linaclotide

\section{Background}

Colonoscopy is considered as the diagnostic gold standard for early detection of colorectal lesions, such as adenoma, polyp, and early colorectal cancer, which has shown great potential to reduce colorectal cancer incidence by removal of these lesions[1]. However, adequate bowel preparation is an essential factor for detection of colorectal neoplasia and to minimize the risk of missed lesions and post-colonoscopy colorectal cancer. Inadequate bowel cleaning will lead to the lower colorectal adenoma rate, longer procedural time of colonoscopy, shorter interval between examinations and increase risk of colonoscopy-related adverse events[2][3]. In clinical practice, up to 10$35 \%$ of all colonoscopy procedures are affected by inadequate bowel preparation[4].

The risk factors of inadequate bowel preparation include chronic constipation, BMI> $25 \mathrm{~kg} / \mathrm{m}^{2}$, colorectal surgery history, and diabetes, et al[5][6]. Identification of risk factors for inadequate bowel preparation will have the potential benefit of screening patients who need more intensive bowel preparation. Previous studies have established three models to predict inadequate bowel preparation[7][8][9]. However, these models are complex and not suitable for clinical practical. In clinical practice, clinicians are more used to assess the risk of bowel preparation failure by peoples' bowel habits, such as stool form and frequency. Some studies have found that stool form and frequency correlate with whole-gut and colonic transit[10][11]. Bristol stool form (BSFS) 1-2[12] and stool frequency $<3$ times per-weekly[13][14] were risk factors for inadequate bowel preparation. However, stool frequency was correlated poorly with all transit[10]. Stool form measured by the BSFS to guide personalized bowel preparation is easy to be identified and can better predict the quality of bowel preparation[15].

Clinical guidelines[7] suggest that additional special regimen or supplemental treatment for people with risk factors of bowel preparation failure. Polyethylene glycol (PEG) is the preferred bowel preparation agent for colonoscopy. It is a highly effective, safe and well-tolerated oral isotonic solution. A previous study found that high-volume PEG or low-volume PEG plus adjunctive agents can provide high-quality bowel 
preparation[16]. However, due to the large volume of bowel preparation, it will reduce patients compliance, acceptability, and willingness to repeat the procedure[17]. Lowvolume PEG plus bisacodyl was confirmed to be an effective strategy for bowel preparation, but it often damaged the mucosa. The ideal bowel preparation should be to clean the colon without damaging the mucosa or causing fluid or electrolyte imbalance. Moreover, it should minimize the discomfort of patients.

Linaclotide is a guanylate cyclase-C agonist which acts on the luminal surface of the entire intestinal epithelium, stimulating secretion of chloride and bicarbonate into the intestinal lumen, resulting in increasing intestinal fluid and increase motility. It is a Food and Drug Administration (FDA) approved medicine that mainly used to treat constipation-predominant irritable bowel syndrome (IBS-C) and chronic idiopathic constipation[18]. A previous study found that single-dose Linaclotide could achieve intestinal cleaning similar to 2L PEG before capsule endoscopy[19]. Another study showed that 2L PEG plus single-dose linaclotide was an effective and well tolerated bowel preparation regimen than 4L PEG[20]. These studies showed that linaclotide can be used as an effective adjunctive agent to provide high-quality bowel preparation. However, no study has evaluated the effect in patients with high-risk factors of inadequate bowel preparation.

The purpose of this study was to evaluate the effect of using linaclotide as an adjunctive agent with 3L PEG for bowel preparation based on Bristol stool form (BSFS).

\section{Methods}

\section{Patients' subjects}

This was a prospective, single-blinded, randomized, controlled study. Adult outpatients (aged from 18 to 80 ) who underwent colonoscopy were enrolled in Tongji Hospital. Exclusion criteria were as follows: (1) history of colorectal surgery; (2) severe heart failure (New York Heart class II-IV); (3) chronic renal failure (stage 2-5) or mental disorder; (4) history of taking linaclotide within 7 days or known linaclotide allergy; (5) patients who were participating in other clinical trials; (6) patients who did 
not provide written informed consent. This study was performed with the approval of the Ethical Committees in accordance with the Declaration of Helsinki, and the informed consents were obtained. The study was registered at Chinese ClinicalTrials.gov (ChiCTR2100050437, 27/8/2021)

\section{Preparation regimens}

At the beginning of enrollment, each patient reported the main stool form he/she defecated in last 7 days according to the BSFS chart. At the time of the colonoscopy appointment, patients with BSFS 1-2 were randomized into either group A or B, and patients with BSFS 3-7 were assigned to group C.

3L PEG was a routine bowel preparation protocol for patients who underwent colonoscopy in our endoscopic center. Patients assigned to group A and C received routine bowel preparation protocol (3L PEG). At 10:00 pm before colonoscopy, two sachets of PEG were dissolved in 2L warm water and were drunk within 1h. At 5:00 am on the day of colonoscopy, another sachet was dissolved in 1L warm water and was drunk within 30 minutes. Patients in group B were asked to take single-dose Linaclotide (290ug) at 8:00 pm the day before colonoscopy and take 3L PEG the same as patients in group A and C. All colonoscopies were performed at 9:00-12:00 am. All patients were provided with written instructions describing the dietary restrictions and the method of bowel preparation.

\section{Outcome measures}

The primary outcome measure was quality of bowel preparation assessed using the Ottawa Bowel Scale (Ottawa) score[21]. The preparation of each colonic segment, divided into the right colon (cecum and ascending colon), the mid colon (transverse and descending colon) and rectosigmoid colon was rated from 0 to 4 . The worst cleanliness score was 4 points. The volume of colonic fluid was rated from 0 to 2 for the entire colon. The maximum volume of fluid score was 2 points. A summary score was then obtained from the individual parameters. We defined each segment Ottawa score $\leq 1$ 
and the total score $\leq 4$ as high-quality bowel preparation, each segment Ottawa score $\leq$ 2 and the total score $\leq 6$ as satisfactory bowel preparation, and each segment Ottawa score $\geq 3$ or the total score $\geq 7$ as failure bowel preparation.

Secondary outcome measure included the polyp detection rate (PDR), patients' acceptability and tolerability, and incidence of adverse events.

\section{Statistical analysis}

In our endoscopic center, the rate of adequate bowel preparation in patients with BSFS 1-2 was about $60 \%$. Based on assumptions of $\alpha=0.05$ and $\beta=0.1$, we calculated that at least 105 patients in each group were need to detect a statistically significant difference between group A and group B with a two-tailed. Achieving a 1:1:1 ratio in 3 groups, we estimated that a total of 315 patients would be adequate to detect a significant difference in the primary endpoint.

SPSS 26.0 was used for the statistical analysis (SPSS, Chicago, IL). Continuous variables were expressed as means with standard deviation (SD) and analyzed using one-way ANOVA, and t-test. Categorical variables were analyzed using the person chisquare test. A $P$-value $<0.05$ was considered statistically significant.

\section{Results}

\section{Baseline characteristics}

A total of 315 patients who underwent colonoscopy were enrolled. 210 patients with BSFS 1-2 were randomized into either group A $(n=105)$ or group $B(n=105)$. Patients with BSFS 3-7 were enrolled into group $(n=105)$. A total of 7 patients cancelled their appointments after randomization. Finally, there were 102, 104 and 102 patients going colonoscopy in group A, B and C, respectively (Fig 1). There were no significant differences among 3 groups upon the baseline characteristics, except that the proportion of female patients $(P=0.000)$ and the proportion of patients with constipation $(P=0.000)$ in group A and B were both much higher than those in group C (Additional file 1: Table S1). 


\section{Quality of bowel preparation}

Bowel preparation quality was scored and summarized in Additional file 2: Table S2.

Compared with group A, patients in group B and C obtained significantly lower Ottawa scores at each colon segment $\left(P_{\mathrm{AB}}<0.05, P_{\mathrm{AC}}<0.05\right)$. There was no significantly difference between group $\mathrm{B}$ and group $\mathrm{C}$ at each colon segment $\left(P_{\mathrm{BC}}>0.05\right)$. The volume of colonic fluid score for the entire colon in group B and group C was significantly lower than that in group $\mathrm{A}\left(1.5 \pm 0.6 \mathrm{vs} 1.2 \pm 0.5, P_{\mathrm{AB}}=0.001,1.5 \pm 0.6 \mathrm{vs}\right.$ $\left.1.0 \pm 0.6, P_{\mathrm{AC}}=0.000\right)$. The volume of colonic fluid score for the entire colon in group $\mathrm{C}$ was significantly lower than that in group $\mathrm{B}\left(1.2 \pm 0.5\right.$ vs $\left.1.0 \pm 0.6, P_{\mathrm{BC}}=0.027\right)$. Total Ottawa scores evaluated of patients in group B and C were significantly lower than that in group $\mathrm{A}\left(5.9 \pm 1.9\right.$ vs $4.8 \pm 1.5, P_{\mathrm{AB}}=0.000,5.9 \pm 1.9$ vs $\left.4.5 \pm 2.1, P_{\mathrm{AC}}=0.000\right)$, However, there was no significantly difference between group B and group C (5.9 \pm 1.9 vs $4.5 \pm 2.1$ vs $\left.4.5 \pm 2.1, P_{\mathrm{BC}}=0.438\right)$.

The satisfactory bowel preparation rate in group B and C were significantly higher than group A $\left(77.9 \%\right.$ vs $61.8 \%, P_{\mathrm{AB}}=0.049,81.3 \%$ vs $\left.61.8 \%, P_{\mathrm{AC}}=0.012\right)$. There was no significantly difference between group $\mathrm{B}$ and group $\mathrm{C}\left(77.9 \%\right.$ vs $\left.81.3 \%, P_{\mathrm{BC}}=0.564\right)$. Furthermore, the high-quality bowel preparation rate in group B also was significantly higher than group $\mathrm{A}\left(21.2 \%\right.$ vs $\left.9.8 \%, P_{\mathrm{AB}}=0.025\right)$, which is similar with group $\mathrm{C}(21.2 \%$

\section{Outcomes of colonoscopy}

The colorectal polyp detection rate (PDR) in group B and C were significantly higher than that in group A $\left(32.7 \%\right.$ vs $15.7 \%, P_{\mathrm{AB}}=0.004,36.3 \%$ vs $\left.15.7 \%, P_{\mathrm{AC}}=0.001\right)$. The colorectal polyp detection rate was similar between group B and C (32.7\% vs $36.3 \%$, $\left.P_{\mathrm{BC}}=0.463\right)$. Considering the size of polyps, there were no significantly difference among 3 groups $(P>0.05)$. Considering the location of colorectal polyps, the PDR of rectosigmoid was significantly higher in group B and C than group A $(25.0 \%$ vs $8.8 \%$, $P_{\mathrm{AB}}=0.002,33.3 \%$ vs $\left.8.8 \%, P_{\mathrm{AC}}=0.000\right)$, in other segments were similar $(P>0.05)$ 
(Additional file 3: Table S3).

\section{Patients' acceptability and complications}

The acceptability and complications are presented in Additional file 4: Table S4. The acceptability was similar between group A, group B and group $\mathrm{C}(P=0.895)$. The complications included nausea, vomiting, bloating and abdominal pain. There were not significantly different among 3 groups $(P=0.116)$.

\section{Discussion}

The bowel preparation quality is an essential factor affecting the quality of colonoscopy examination, such as colorectal adenomas detection rate, duration of colonoscopy, interval between taking colonoscopy examinations and risk of colonoscopy related adverse events. In our study, compared with the routine scheme (3L PEG), the intensive scheme (3L PEG + Linaclotide) obtained lower Ottawa scores in terms of the both whole colon and each colon segment in patients with BSFS 1-2, and achieved the similar bowel preparation quality to routine scheme (3L PEG) in patients with BSFS 3-7.

Clinical guidelines[7] recommend that a special regimen or supplemental treatment for bowel preparation in patients with high risk factors. Low-volume PEG (2L) plus adjunctive agents (such as bisacodyl, ascorbate and magnesium citrate) can provide high-quality bowel preparation. However, these adjunctive agents are limited by their side effects and contraindications. Linaclotide has high security, few clinical complications and minimal systemic absorption and can increase active secretion of small intestinal juices and colonic transit speed. We found that the Ottawa scores for the total and segment colons, the bowel preparation efficiency of 3L PEG + Linaclotide were superior to that of $3 \mathrm{~L}$ PEG in patients with BSFS 1-2 $(P=0.000)$, and which is similar in patients with BSFS 3-7 $(P=0.438)$. The rate of adequate bowel preparation in group B was similar to that in group C $(77.9 \%$ vs $81.3 \%, P=0.564)$, and superior than 
preparation quality of $2 \mathrm{~L}$ PEG + linaclotide were superior to that of $2 \mathrm{~L}$ PEG $(91.4 \%$ vs $77.0 \%, P=0.001)[20]$. It was comparable to the $70 \%-91 \%$ efficacy rate reported for other adjunctive agents combined with low-volume[22][23]. We demonstrated the ability of 3L PEG + Linaclotide in improving bowel preparation quality before colonoscopy, and achieve the similar results as using other adjunctive agents. We consider that PEG works as an osmotic laxative and Linaclotide is a GC-C receptor agonist, the combination of these two drugs may promote their individual effect on bowel preparation through two different mechanisms.

The adenoma detection rate (ADR) has emerged as the most important quality measure in colonoscopy[24]. The risk of missing colorectal adenomas is expected to be particularly high in case of inadequate bowel preparation, a problem commonly encountered in routine practice[25]. In our study, total colorectal PDR of group B were higher than that of group A $(32.7 \%$ vs $15.7 \%, P=0.004)$, which was similar to that of group C $(32.7 \%$ vs $36.3 \%, P=0.463)$. However, the polyps detection rate was lower than other researches $(37 \%-43 \%)[26][27]$. It may be that in the practice of colonoscopy, the withdrawal time of colonoscopy by endoscopists was less than 6 minutes, which is recommended by the guidelines. Considering the location of polyp, we found that the rectosigmoid colon PDR of group B were higher than that of group A $(25.0 \%$ vs $8.8 \%$, $P=0.002$ ). Previous studies also showed that a significant miss rates of adenomas located in the proximal if bowel preparation is not adequate[3][25]. No matter how, improving the bowel preparation quality may significantly improve the PDR, especially in the rectosigmoid.

The patients' tolerability and willingness to repeat colonoscopies depend on the complications of the bowel reparation regimen. In our study, the incidence of complications (such as nausea, vomiting and abdominal distension) were similar among three groups. According to some clinical trials[28][29], Linaclotide is effective, safe, and well tolerated in IBS-C and chronic constipation patients.

There are some limitations in our study. First, this is a single center and small sample size study, which may limit the generalizability of these results. Secondly, we calculated 
the polyp detection rate (PDR) instead of the adenoma detection rate (ADR). Thirdly, the routine bowel preparation protocol is $3 \mathrm{~L}$ PEG in our center, and the pre-experiment found that the rate of adequate bowel preparation in patients with BSFS 1-2 was about $60 \%$. Therefore, our study was designed based on the routine bowel preparation protocol (3L PEG) plus Linaclotide. However, previous studies used 2L PEG plus additional as an intensive bowel preparation. In the future, we will further explore the bowel preparation quality between 2L PEG plus Linaclotide and 3L PEG bowel preparation protocols.

\section{Conclusions}

In summary, our study implies that 3L PEG plus 290ug Linaclotide can significantly improve the quality of bowel preparation and the colorectal polyp detection rate, especially the proximal colon polyps. 3L PEG plus 290ug Linaclotide is an effective and well-tolerated bowel preparation regimen.

\section{Additional files}

Additional file 1: Table S1. Baseline characteristics of the study patients in intention to treat analysis. (DOCX 16kb)

Additional file 2: Table S2. bowel preparation quality (per-protocol analysis). (DOCX $16 \mathrm{~kb})$

Additional file 3: Table S3. Outcomes of colonoscopy (per-protocol analysis). (DOCX $16 \mathrm{~kb})$

Additional file 4: Table S4. Patients' acceptability and tolerability in intention to treat analysis. (DOCX 15kb)

\section{Abbreviations}

PEG: polyethylene glycol; BSFS: Bristol stool form; PDR: polyp detection rate; ADR: adenoma detection rate; FDA: Food and Drug Administration; IBS-C: constipationpredominant irritable bowel syndrome; Ottawa: Ottawa Bowel Scale; SD: standard 


\section{Acknowledgement}

The authors thank the endoscopists at the Tongji Hospital who helped perform the colonoscopy.

\section{Author contributions} and writing. G.W. assisted in study design and implemented. S.X., J.W., W.Z. and M.Z. assisted with data collections and analysis. M.L. and D.T. assisted in study design and supervised. J.L. assisted with study design, implemented the study protocol, and revision of the manuscript. All authors read and approval the final manuscript.

\section{Funding}

The research was supported by grans form the National Natural Science Foundation of China No. 81672392 and Provincial Natural Science Foundation of Hubei No. 2015 CFA071.

\section{Availability of data and materials}

The data analyzed for this study can be accessible from the corresponding author on was registered at Chinese ClinicalTrials.gov (ChiCTR2100050437, 27/8/2021)

\section{Consent for publication}

Not applicable. 


\section{Competing interests}

293 The authors declare that they have no conflicts of interest.

\section{Author details}

Department of Gastroenterology, Tongji Hospital of Tongji Medical College, Huazhong university of Science and Technology, Wuhan 430030 Hubei Province, China

\section{References}

1. Sung JJ, Ng SC, Chan FK, et al. An updated Asia Pacific Consensus Recommendations on colorectal cancer screening. Gut. 2015; 64(1):121-132.

2. Froehlich F, Wietlisbach V, Gonvers JJ, et al. Impact of colonic cleansing on quality and diagnostic yield of colonoscopy: the European Panel of Appropriateness of Gastrointestinal Endoscopy European multicenter study. Gastrointest Endosc. 2005; 61(3):378-384.

3. Niedermaier T, Amitay EL, Gies A, et al. Impact of Inadequate Bowel Cleansing on Colonoscopic Findings in Routine Screening Practice. Clin Transl Gastroenterol. 2020; 11(4):e00169.

4. Johnson DA, Barkun AN, Cohen LB, et al. Optimizing adequacy of bowel cleansing for colonoscopy: recommendations from the US Multi-Society Task Force on Colorectal Cancer. Am J Gastroenterol. 2014; 109(10):1528-1545. 5. Mahmood S, Farooqui SM, Madhoun MF. Predictors of inadequate bowel preparation for colonoscopy: a systematic review and meta-analysis. Eur $\mathrm{J}$ Gastroenterol Hepatol. 2018; 30(8):819-826.

6. Gandhi K, Tofani C, Sokach C, et al. Patient characteristics associated with quality of colonoscopy preparation: a systematic review and meta-analysis. Clin Gastroenterol Hepatol. 2018; 16: $357-369 . e 10$ 7. Hassan C, East J, Radaelli F, et al. Bowel preparation for colonoscopy: European 
2019; 51(8):775-794.

8. Dik VK, Moons LM, Hüyük M, et al. Predicting inadequate bowel preparation for colonoscopy in participants receiving split-dose bowel preparation: development and validation of a prediction score. Gastrointest Endosc. 2015; 81(3):665-672.

9. Gimeno-García A, Baute J, Hernandez G, et al. Risk factors for inade- quate bowel preparation: a validated predictive score. Endoscopy. 2017; 49: 536 - 543

10. Törnblom H, Van Oudenhove L, Sadik R, et al. Colonic transit time and IBS symptoms: what's the link? Am J Gastroenterol. 2012; 107(5):754-760.

11. Malhotra A, Shah N, Depasquale J, et al. Use of Bristol Stool Form Scale to predict the adequacy of bowel preparation - a prospective study. Colorectal Dis. 2016; 18(2):200-204.

12. Guo X, Shi X, Kang X, et al. Risk Factors Associated with Inadequate Bowel Preparation in Patients with Functional Constipation. Dig Dis Sci. 2020; 65(4):10821091.

13. Lee DW, Koo JS, Kang S, et al. Association between bowel habits and quality of bowel preparation for colonoscopy. Medicine (Baltimore). 2017; 96(29):e7319.

14. Paik N, Kim ER, Kim TJ, et al. Usefulness of Personal Bowel Habits as a Predictive Factor for Inadequate Bowel Preparation for Colonoscopy: A Prospective Questionnaire-Based Observational Study. Gut Liver. 2019; 13(2):169-175.

15. Saad RJ, Rao SS, Koch KL, et al. Do stool form and frequency correlate with whole-gut and colonic transit? Results from a multicenter study in constipated individuals and healthy controls. Am J Gastroenterol. 2010; 105(2):403-411.

16. Mohamed R, Hilsden RJ, Dube C, et al. Split-Dose Polyethylene Glycol Is Superior to Single Dose for Colonoscopy Preparation: Results of a Randomized Controlled Trial. Can J Gastroenterol Hepatol. 2016; 2016:3181459.

17. Spadaccini M, Frazzoni L, Vanella G, et al. Efficacy and Tolerability of High- vs Low-Volume Split-Dose Bowel Cleansing Regimens for Colonoscopy: A Systematic Review and Meta-analysis. Clin Gastroenterol Hepatol. 2020; 18(7):1454-1465.e1414. 18. Mascarenhas-Saraiva MJ, Mascarenhas-Saraiva M. Effectiveness and tolerability 
of linaclotide in the treatment of IBS-C in a "real-life" setting: Results from a Portuguese single-center study. Neurogastroenterol Motil. 2019; 31(2):e13508. 19. Stein DJ, Copland A, McDaniel D, et al. Single-Dose Linaclotide Is Equal in Efficacy to Polyethylene Glycol for Bowel Preparation Prior to Capsule Endoscopy. Dig Dis. 2019; 37(4):297-302.

20. Zhang M, Zou W, Xu C, et al. Polyethylene glycol combined with linaclotide is an effective and well-tolerated bowel preparation regimen for colonoscopy: an endoscopist-blinded, randomized, controlled trial. Eur J Gastroenterol Hepatol. 2021. 21. Rostom A, Jolicoeur E. Validation of a new scale for the assessment of bowel preparation quality. Gastrointest Endosc. 2004; 59(4):482-486.

22. Spada C, Cesaro P, Bazzoli F, et al. Evaluation of Clensia( $\left({ }^{\circledR}\right)$, a new low-volume PEG bowel preparation in colonoscopy: Multicentre randomized controlled trial versus

23. Kim ES, Kim KO, Jang BI, et al. Comparison of 4-L Polyethylene Glycol and 2L Polyethylene Glycol Plus Ascorbic Acid in Patients with Inactive Ulcerative Colitis. Dig Dis Sci. 2017; 62(9):2489-2497.

24. Rex DK. Polyp detection at colonoscopy: Endoscopist and technical factors. Best Pract Res Clin Gastroenterol. 2017; 31(4):425-433.

25. Zhao S, Wang S, Pan P, et al. Magnitude, Risk Factors, and Factors Associated With Adenoma Miss Rate of Tandem Colonoscopy: A Systematic Review and Metaanalysis. Gastroenterology. 2019; 156(6):1661-1674.e1611.

26. Sey MSL, Von Renteln D, Sultanian R, et al. A multi-centre randomized controlled trial comparing two bowel cleansing regimens for colonoscopy after failed bowel preparation. Clin Gastroenterol Hepatol. 2021.

27. Andreozzi P, De Nucci G, Bezzio C, et al. Comparison of asymmetric (low morning-dose) and standard split-dose regimen of PEG plus bisacodyl for bowel preparation: A randomized controlled trial. Dig Liver Dis. 2019; 51(6):837-842.

28. Serrano-Falcón B, Delgado-Aros S, Mearin F, et al. Clinical response to linaclotide at week 4 predicts sustained response in irritable bowel syndrome with 
378 constipation and improvements in digestive and extra-digestive symptoms. Therap Adv 379 Gastroenterol. 2019; 12:1756284819857358.

380 29. Fukudo S, Miwa H, Nakajima A, et al. High-dose linaclotide is effective and safe 381 in patients with chronic constipation: A phase III randomized, double-blind, placebo382 controlled study with a long-term open-label extension study in Japan. 383 Neurogastroenterol Motil. 2019; 31(1):e13487. 


\section{Figures}

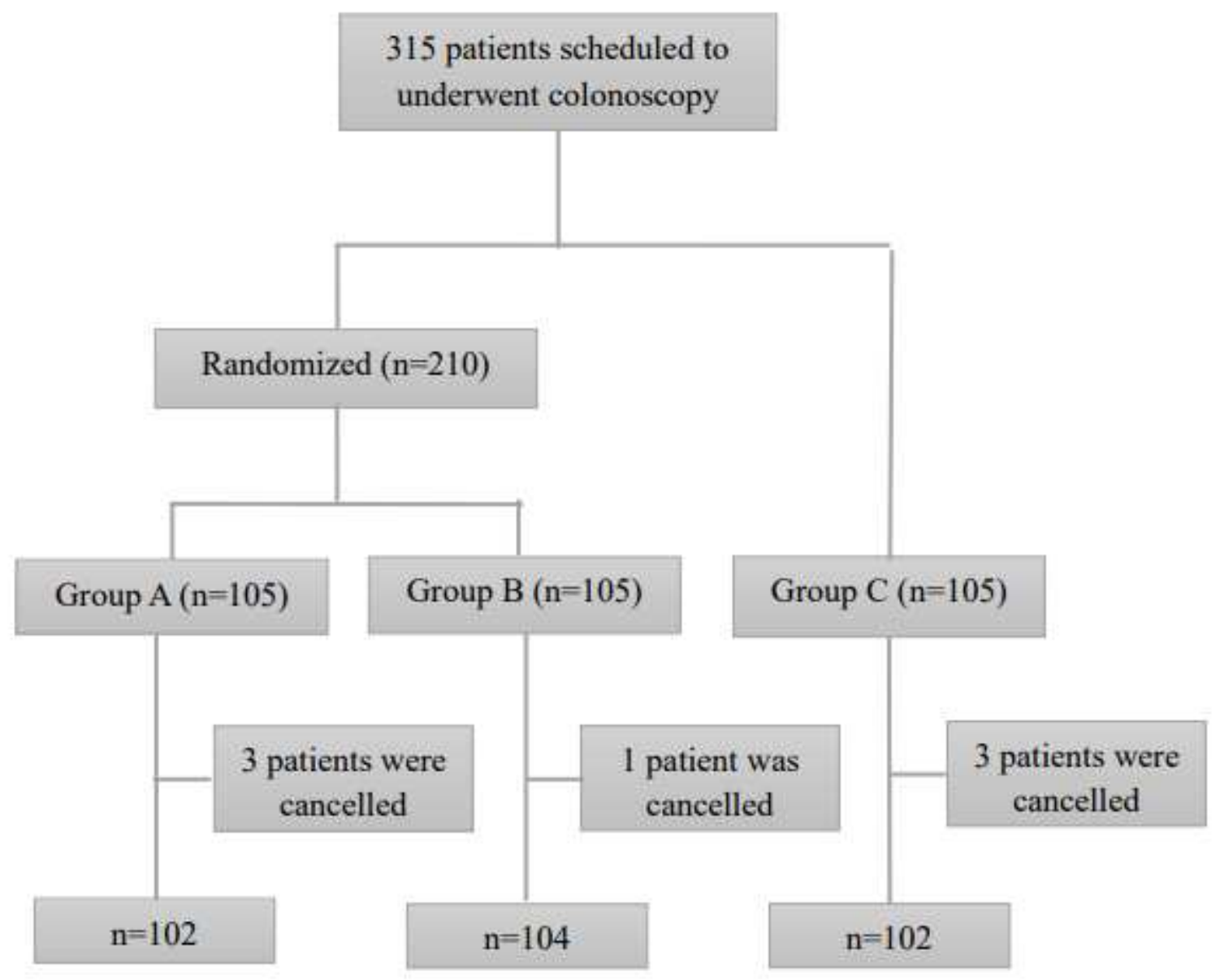

\section{Figure 1}

Flowchart of the study

\section{Supplementary Files}

This is a list of supplementary files associated with this preprint. Click to download.

- Additionalfile1.docx

- Additionalfile2.docx

- Additionalfile3.docx

- Additionalfile4.docx 